\title{
MOHAMED MAGHFOUL Sur la forte $K$-moyennabilité d'un groupoïde
}

Annales de la faculté des sciences de Toulouse $\sigma^{e}$ série, tome 10 , $\mathrm{n}^{\circ} 4$ (2001), p. 683-699

<http://www.numdam.org/item?id=AFST_2001_6_10_4_683_0>

(C) Université Paul Sabatier, 2001, tous droits réservés.

L'accès aux archives de la revue «Annales de la faculté des sciences de Toulouse » (http://picard.ups-tlse.fr/ annales/) implique l'accord avec les conditions générales d'utilisation (http://www.numdam.org/conditions). Toute utilisation commerciale ou impression systématique est constitutive d'une infraction pénale. Toute copie ou impression de ce fichier doit contenir la présente mention de copyright.

\section{Numdam}

Article numérisé dans le cadre du programme Numérisation de documents anciens mathématiques http://www.numdam.org/ 


\title{
Sur la forte $K$-moyennabilité d'un groupoïde ${ }^{(*)}$
}

\author{
MOHAMED MAGHFOUL ${ }^{(1)}$
}

RÉsumé. - Nous discutons quelques propriétés de la forte $K$ moyennabilité pour les groupoïdes et nous l'appliquons aux suites exactes en théorie de Kasparov équivariante.

Abstract. - We discuss some properties of the strong $K$-amenability for groupoids and we apply this to the exact sequences in equivariant Kasparov's theory.

\section{Introduction.}

En étudiant le comportement du bifoncteur de Kasparov équivariant vis-à-vis des suites exactes de $C^{*}$-algèbres, nous avons introduit dans [12] une notion de forte $K$-moyennabilité pour les groupes. Nous avons montré que pour les groupes $G$ possédant cette propriété, le bifoncteur $K K_{G}$ est semi-exact sous des hypothèses de relèvement complètement positif ou de nucléarité en $K$-théorie équivariante.

La théorie de Kasparov équivariante a été généralisée au cas des actions d'un groupoïde [7]. Ceci fournit un cadre plus général dans lequel la $K K$-théorie équivariante s'explique mieux et peut avoir de nombreuses applications $([16],[17])$.

Dans cet article, nous étudions quelques propriétés de la forte $K$ moyennabilité pour un groupoïde. Nous appliquons cette notion au problème d'existence de suites exactes a six termes en théorie de Kasparov équivariante par rapport à des groupoïdes et nous obtenons le résultat suivant:

(*) Reçu le 29 novembre 1999, accepté le 5 juin 2002

(1) Faculté des Sciences, Département de Mathématiques et Informatique, Université Ibn Tofail, Kénitra, Maroc. E-mail: mmaghfoul@mailcity.com 


\section{Mohamed Maghfoul}

THÉORÈmE 0.1. - Soient $G$ un groupoïde localement compact fortement $K$-moyennable et $0 \longrightarrow J \stackrel{j}{\longrightarrow} B \stackrel{q}{\longrightarrow} B / J \longrightarrow 0$ une suite exacte $G$ équivariante de $G$-algèbres avec $B$ séparable. Soit $A$ une $G$-algèbre séparable.

- Si l'application $q$ admet un relèvement complètement positif $C(X)$ linéaire (non nécessairement équivariant) de norme 1 , ou si $J, B$ et $B / J$ sont $C(X)$-nucléaires en $K$-théorie équivariante, on a les suites exactes hexagonales suivantes:

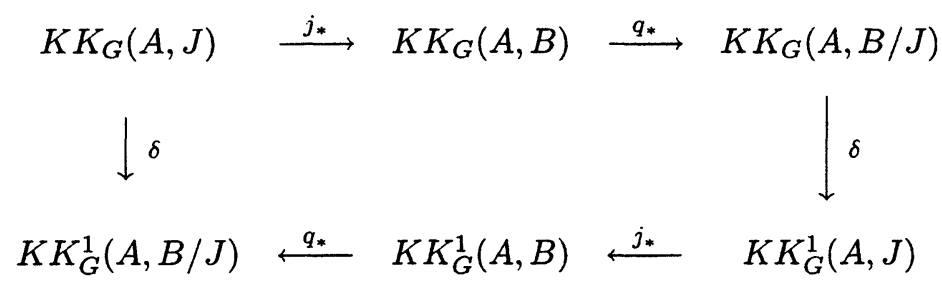

et

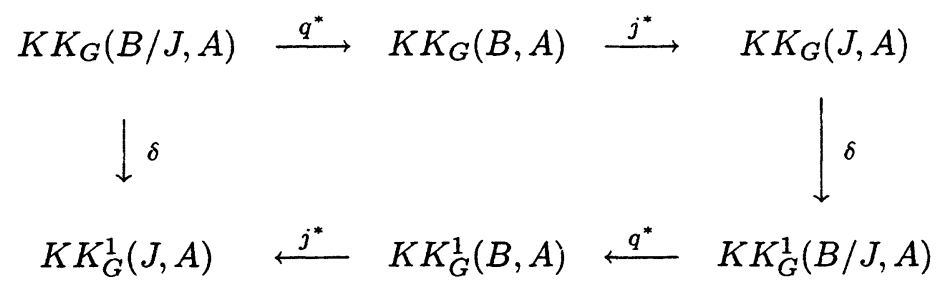

- Si $A$ est $C(X)$-nucléaire en $K$-théorie équivariante, on a la suite exacte hexagonale:

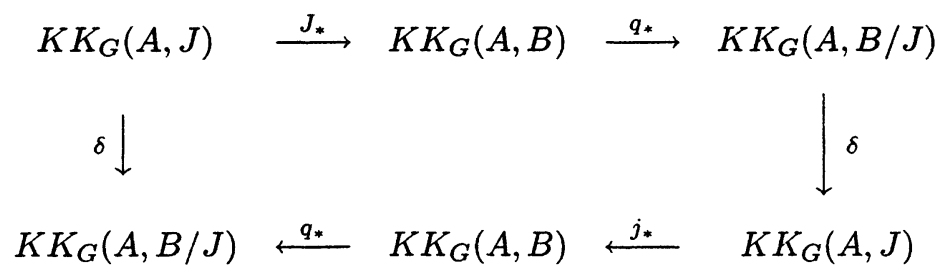


Ce théorème généralise les résultats de [12] et [3] ainsi que certains résultats partiels de [16] et [17] dans le cas des groupoïdes.

Remerciements. - Je tiens à exprimer mes remerciements au Professeur Georges Skandalis pour les discussions enrichissantes que j'ai pu avoir avec lui pendant la réalisation de ce travail.

\section{Préliminaires et notations}

Pour la définition et les propriétés de la $K K$-théorie équivariante par rapport à un groupoïde, nous renvoyons à [7]. Les notations et la terminologie concernant ce bifoncteur sont essentiellement celles de ([10],[7],[12]).

Si $X$ est un espace localement compact, on note $C(X)$ l'espace des fonctions continues sur $X$ nulles à l'infini et $C_{c}(X)$ celui des fonctions continues à support compact.

Soit $G$ un groupoïde localement compact ( $\sigma$ - compact) de base $X$ et muni d'un système de Haar $\lambda=\left\{\lambda^{x}, x \in X\right\}$ (cf.[13]). On note $L^{2}(G, \lambda)$ le $C(X)$-module hilbertien $G$-équivariant complété de $C_{c}(G)$ pour le produit scalaire:

$$
\langle\xi, \eta\rangle(x)=\int_{G^{x}}\langle\xi(g), \eta(g)\rangle(x) d \lambda^{x}(g)
$$

et sur lequel $G$ agit par la translation à gauche $\lambda^{G}$. L'action de $C(X)$ sur $L^{2}(G, \lambda)$ est telle que $\xi f(g)=\xi(g) f(r(g))$ pour tous $\xi \in L^{2}(G, \lambda)$ et $f \in C(X)$. Soient $B$ une $G$-algèbre (séparable), $\mathcal{E}$ un $B$-module hilbertien (dénombrablement engendré) équivariant et $C_{c}(G, \mathcal{E})$ l'espace des fonctions continues à support compact de $G$ à valeurs dans $\mathcal{E}$. Sur $C_{c}(G, \mathcal{E})$ on considère les opérations suivantes:

$$
\begin{aligned}
& -(f b)(g)=f(g) \alpha_{g}\left(b_{s(g)}\right) \\
& \text { - } g(f)\left(g_{1}\right)=g\left(f\left(g^{-1} g_{1}\right)\right)
\end{aligned}
$$

pour tous $g, g_{1} \in G ; b \in B ; f \in C_{c}(G, \mathcal{E})$. L'application:

$$
x \mapsto\left\langle f_{1}, f_{2}\right\rangle_{x}=\int_{G_{x}} \alpha_{g^{-1}}\left(\left\langle f_{1}(g), f_{2}(g)\right\rangle_{r(g)}\right) d \lambda_{x}(g)
$$

(pour $\left.f_{1}, f_{2} \in C_{c}(G, \mathcal{E})\right)$ appartient à $C\left(X, B^{\sharp}\right)$ et donc définit un élément de $B$ ([7] lemmes 7.1.1 et 7.1.2) qu'on note $\left\langle f_{1}, f_{2}\right\rangle$ avec $\lambda_{x}$ est la mesure image de $\lambda^{x}$ par l'application qui à $g$ associe $g^{-1}$. On note $L^{2}(G, \mathcal{E})$ le $B$-module hilbertien $G$-équivariant complété de $C_{c}(G, \mathcal{E})$ pour la norme

$$
\|f\|=\operatorname{Sup}_{x \in X}\left\|\langle f, f\rangle_{x}\right\|^{\frac{1}{2}} .
$$


L'application: $f(g) \otimes \xi \mapsto f(g) \cdot \xi_{r(g)}$ définit un isomorphisme entre $L^{2}(G) \otimes_{C(X)} \mathcal{E}$ et $L^{2}(G, \mathcal{E})$. Remarquons que les éléments de $L^{2}(G, \mathcal{E})$ sont des fonctions sur $G$ telles que $f(g) \in \mathcal{E}_{r(g)}$ pour tout $g \in G$. Pour $A$ une $C^{*}$-algèbre, on note $M(A)$ la $C^{*}$-algèbre des multiplicateurs de $A$.

Dans la suite, toutes les $C^{*}$-algèbres et tous les modules hilbertiens sont supposés $Z / 2 Z$-gradués et dénombrablement engendrés. Tous les groupoïdes sont supposés séparés séparables.

\section{Forte $K$-moyennabilité}

Dans cette section, nous discutons quelques propriétés de la forte $K$ moyennabilité pour un groupoïde.

DÉfINITION 2.1. - Soit $G$ un groupoïde localement compact de base $X$ et muni d'un système de Haar. Soient $\mathcal{H}_{G}^{(i)}=L^{2}(G, \lambda) \otimes l^{2}(\mathbb{N}) ; i=0,1$, $\mathcal{H}_{G}=\mathcal{H}_{G}^{(0)} \oplus \mathcal{H}_{G}^{(1)}$. On dira que $G$ est fortement $K$-moyennable, s'il existe $F \in \mathcal{L}\left(\mathcal{H}_{G}\right)^{(1)}$ tel que $\left(\mathcal{H}_{G}, F\right)$ soit de classe 1 dans $K K_{G}(C(X), C(X))$.

Ceci équivaut à dire qu'il existe un sous- $C(X)$-module hilbertien $H, G$-invariant, de $\mathcal{H}_{G}$ et $F \in \mathcal{L}(H)^{(1)}$ tel que $\left(\mathcal{H}_{G}, F\right)=1$ dans $K K_{G}(C(X), C(X))$ [12]. Le groupoïde $G$ agit sur $\mathcal{H}_{G}$ par $\lambda^{G} \otimes 1$. Si $G$ est un groupe, alors $X=\{e\}$ et $K K_{G}(C(X), C(X))=K K_{G}(\boldsymbol{C}, \boldsymbol{C})$, on retrouve ainsi la définition de la forte $K$-moyennabilité pour les groupes [12]. Soit $G$ un groupoïde localement compact agissant proprement et par isométrie affines sur un champ continu $H=\left(H_{x}\right)_{x \in X}$ d'espaces affines euclidiens où $X=G^{(0)}$. Dans ([11], [16]) on construit pour $G$ vérifiant ces hypothèses une $G$-algèbre $\mathcal{A}(H)$ et deux éléments $\eta \in K K_{G}(C(X), \mathcal{A}(H))$ et $D \in K K_{G}(\mathcal{A}(H), C(X))$. Soit $\gamma=\eta \otimes_{\mathcal{A}(H)} D$ : le produit de Kasparov interne. Puisque l'action de $G$ est propre, le théorème de stabilisation montre que le $\mathcal{A}(H)$-module hilbertien $\mathcal{A}(H)$ représentant $\eta$ est un sous-module $G$ invariant de $\mathcal{H}_{G} \otimes_{C(X)} \mathcal{A}(H)$ et donc (et pour les mêmes raisons) le $C(X)$ module représentant $\gamma$ est un sous- $C(X)$-module $G$-invariant de $\mathcal{H}_{G}$. On montre (voir [16]) que $\gamma=1$ dans $K K_{G}(C(X), C(X)$ ). Donc $G$ est fortement $K$-moyennable. En particulier, si $G$ est topologiquement moyennable [1], alors $G$ est fortement $K$-moyennable.

Proposition 2.2. - Soient $G_{1}$ et $G_{2}$ deux groupoïdes localement compacts munis de systèmes de Haar. Si $G_{1}$ et $G_{2}$ sont fortement $K$ moyennables, alors $G_{1} \times G_{2}$ est fortement $K$-moyennable.

Démonstration. - Supposons que $G_{1}$ et $G_{2}$ sont fortement $K$ moyennables. Soit $F_{i} \in \mathcal{L}\left(\mathcal{H}_{G_{i}}\right)$ tel que $\left(\mathcal{H}_{G_{i}}, F_{i}\right)$ soit de classe 1 dans 
$K K_{G_{i}}\left(C\left(X_{i}\right), C\left(X_{i}\right)\right)(i=1,2)$. Soit

$$
p_{i}^{*}: K K_{G_{i}}\left(C\left(X_{i}\right), C\left(X_{i}\right)\right) \longrightarrow K K_{G_{1} \times G_{2}}\left(C\left(X_{1} \times X_{2}\right), C\left(X_{1} \times X_{2}\right)\right)
$$

l'homomorphisme associé à la projection $p_{i}: G_{1} \times G_{2} \longrightarrow G_{i}, i=1,2$. Le produit de Kasparov de $p_{1}^{*}\left(\left(\mathcal{H}_{G_{1}}, F_{1}\right)\right)$ par $p_{2}^{*}\left(\left(\mathcal{H}_{G_{2}}, F_{2}\right)\right)$ est de la forme: $\left(\mathcal{H}_{G_{1} \times \mathcal{H}_{G_{2}}}, F\right)$ et comme $p_{i}^{*}\left(\left(\mathcal{H}_{G_{i}}, F_{i}\right)\right)=1,(i=1,2)$, on a $\left(\mathcal{H}_{G_{1} \times \mathcal{H}_{G_{2}}}, F\right)=$ 1 dans $K K_{G_{1} \times G_{2}}\left(C\left(X_{1} \times X_{2}\right), C\left(X_{1} \times X_{2}\right)\right)$.

Proposition 2.3. - Soient $G_{1}$ et $G_{2}$ deux groupoïdes localement compacts munis de systèmes de Haar et $f: G_{1} \longrightarrow G_{2}$ un homomorphisme propore. Si $G_{2}$ est fortement $K$-moyennable, alors il en est de même pour $G_{1}$.

CoROllaire 2.4. - Soient $G$ un groupoïde localement compact et $H \subset$ $G$ un sous-groupoïde fermé. Si $G$ est fortement $K$-moyennable, alors il en est de même pour $H$.

Démonstration. - Découle du fait que l'inclusion de $H$ dans $G$ est propre.

La démonstration de la proposition (2.3) nécessite quelques préliminaires.

LEMME 2.5 ([4] proposition 2.4.1). - Soient A une algèbre de Banach involutive, $\pi$ et $\pi^{\prime}$ deux représentations de $A$ dans les modules hilbertiens $\mathcal{E}$ et $\mathcal{E}^{\prime}$. Soit $\xi\left(\right.$ resp. $\xi^{\prime}$ ) un vecteur totalisateur pour $\pi$ (resp. pour $\pi^{\prime}$ ). Si $\langle\pi(x) \xi, \xi\rangle=\left\langle\pi^{\prime}(x) \xi^{\prime}, \xi^{\prime}\right\rangle$ pour tout $x \in A$, il existe un unique unitaire $U$ de $\mathcal{E}$ sur $\mathcal{E}^{\prime}$ transformant $\pi$ en $\pi^{\prime}$ et $\xi$ en $\xi^{\prime}$.

Démonstration. - Pour tous $x, y$ éléments de $A$, on a

$$
\begin{aligned}
\langle\pi(x) \xi, \pi(y) \xi\rangle & =\left\langle\pi\left(y^{*} x\right) \xi, \xi\right\rangle \\
& =\left\langle\pi^{\prime}\left(y^{*} x\right) \xi^{\prime}, \xi^{\prime}\right\rangle \\
& =\left\langle\pi^{\prime}(x) \xi^{\prime}, \pi^{\prime}(y) \xi^{\prime}\right\rangle
\end{aligned}
$$

Comme les $\pi(x) \xi$ ( resp. les $\pi^{\prime}(x) \xi^{\prime}$ ) sont partout denses dans $\mathcal{E}$ (resp. dans $\mathcal{E}^{\prime}$ ), il existe alors un isomorphisme de $\mathcal{E}$ dans $\mathcal{E}^{\prime}$ tel que $U(\pi(x) \xi)=$ $\pi^{\prime}(x) \xi^{\prime}$ pour tout $x \in A$. Montrons que $U$ entrelace les représentations $\pi$ et $\pi^{\prime}$; c'est à dire $U \pi(x)=\pi^{\prime}(x) U$ quelque soit $x$ dans $A$. 
Soit $y \in A$, alors

$$
\begin{aligned}
(U \pi(x))(\pi(y) \xi) & =U(\pi(x y)) \xi \\
& =\pi^{\prime}(x y) \xi^{\prime} \\
& =\pi^{\prime}(x)\left(\pi^{\prime}(y) \xi^{\prime}\right) \\
& =\left(\pi^{\prime}(x) U\right)(\pi(y) \xi) .
\end{aligned}
$$

Comme les $\pi(y) \xi$ sont denses dans $\mathcal{E}$, on a bien $U \pi(x)=\pi^{\prime}(x) U$. D'autre part, pour tout $x \in A$, on a

$$
\begin{aligned}
\left\langle\xi^{\prime}, \pi^{\prime}(x) \xi^{\prime}\right\rangle & =\langle\xi, \pi(x) \xi\rangle \\
& =\langle U \xi, U \pi(x) \xi\rangle \\
& =\left\langle U \xi, \pi^{\prime}(x) \xi\right\rangle .
\end{aligned}
$$

Par conséquent $\xi^{\prime}=U(\xi)$. L'unicité de $U$ se déduit facilement puisque les valeurs de $U$ sont imposées sur $\pi(A) \xi$.

Pour $X$ un espace localement compact et $x \in X$ on note $e_{x}: C(X) \longrightarrow \boldsymbol{C}$ l'évaluation au point $x$. Si $\mathcal{E}$ est un $C(X)$-module hilbertien, on désignera par $\xi_{x}$ l'image de $\xi \in \mathcal{E}$ dans $\mathcal{E}_{x}=\mathcal{E} \otimes_{e_{x}} \boldsymbol{C}$. Pour $\mathcal{E}^{\prime}$ un autre $C(X)$-module hilbertien et $T \in \mathcal{L}\left(\mathcal{E}, \mathcal{E}^{\prime}\right)$, on note $T_{x}$ l'image de $T$ dans $\mathcal{L}\left(\mathcal{E}_{x}, \mathcal{E}_{x}^{\prime}\right)$ définie $\operatorname{par} T_{x}\left(\xi \otimes_{e_{x}} 1\right)=T(\xi) \otimes_{e_{x}} 1$.

Pour $G$ un groupoïde localement compact de base $X$ et muni d'un système de Haar $\lambda$ on note $L^{1}(G, \lambda)$ l'algèbre de Banach involutive complété de $C_{c}(G)$ pour la norme

$$
\|\phi\|=\max \left\{|\phi|_{1},\left|\phi^{*}\right|_{1}\right\}
$$

où

$$
\begin{gathered}
|\phi|_{1}=\sup _{x \in X} \int_{\gamma \in G^{x}}|\phi(\gamma)| \lambda^{x}(\gamma) \\
\phi^{*}(\gamma)=\overline{\phi\left(\gamma^{-1}\right)} .
\end{gathered}
$$

La multiplication est donnée par le produit de convolution:

$$
f * g(\gamma)=\int_{\gamma^{\prime} \in G^{r(\gamma)}} f(\gamma) g\left(\gamma^{\prime-1} \gamma\right) d \lambda^{r(\gamma)}\left(\gamma^{\prime}\right)
$$

Soient $\mathcal{E}$ un $C(X)$-module hilbertien et $\pi$ une action (au sens de [7], 
définition 4.4) de $G \operatorname{sur} \mathcal{E}$. L'action $\pi$ induit une représentation (qu'on notera encore $\pi$ ) de $L^{1}(G, \lambda)$ dans $\mathcal{E}$ définie par

$$
\langle\pi(f) \xi, \eta\rangle(x)=\int_{\gamma \in G^{x}}\left\langle\pi_{\gamma} \xi_{s(\gamma)}, \eta_{r(\gamma)}\right\rangle f(\gamma) d \lambda^{x}(\gamma) .
$$

Nous dirons qu'un vecteur $\xi \in \mathcal{E}$ est totalisateur pour l'action $\pi$ si le sous-espace engendré par $\left\{\pi(f) \xi, f \in L^{1}(G, \lambda)\right\}$ est dense dans $\mathcal{E}$.

LEMME 2.6. - Soient $G$ un groupoïde localement compact, $\mathcal{E}$ et $\mathcal{E}^{\prime}$ deux $C(X)$-modules hilbertiens. Soient $\pi$ (resp. $\pi^{\prime}$ ) une action de $G$ dans $\mathcal{E}$ (resp. dans $\mathcal{E}^{\prime}$ ). Soit $\xi$ (resp. $\left.\xi^{\prime}\right)$ un vecteur totalisateur pour $\pi$ (resp. pour $\left.\pi^{\prime}\right)$. Si, pour tout $\gamma \in G,\left\langle\pi_{\gamma} \xi_{s(\gamma)}, \xi_{r(\gamma)}\right\rangle=\left\langle\pi_{\gamma}^{\prime} \xi_{s(\gamma)}^{\prime}, \xi_{r(\gamma)}^{\prime}\right\rangle$, alors il existe un unique unitaire $U$ de $\mathcal{E}$ sur $\mathcal{E}^{\prime}$ tel que pour tout $\gamma \in G$, on ait :

$$
U_{r(\gamma)}^{*} \pi_{\gamma}^{\prime} U_{s(\gamma)}=\pi_{\gamma} \text { et } U_{s(\gamma)} \xi_{s(\gamma)}=\xi_{s(\gamma)}^{\prime} .
$$

Démonstration. - Notons encore $\pi$ (resp. $\pi^{\prime}$ ) la représentation de $L^{1}(G, \lambda)$ dans $\mathcal{E}$ (resp. $\mathcal{E}^{\prime}$ ) induite par l'action de $G$. Pour tous $x \in X$ et $f \in L^{1}(G, \lambda)$, on a:

$$
\begin{aligned}
\langle\pi(f) \xi, \xi\rangle(x) & =\int_{\gamma \in G^{x}}\left\langle\pi_{\gamma} \xi_{s(\gamma)}, \xi_{r(\gamma)}\right\rangle f(\gamma) d \lambda^{x}(\gamma) \\
& =\int_{\gamma \in G^{x}}\left\langle\pi_{\gamma}^{\prime} \xi_{s(\gamma)}^{\prime}, \xi_{r(\gamma)}^{\prime}\right\rangle f(\gamma) d \lambda^{x}(\gamma) \\
& =\left\langle\pi^{\prime}(f) \xi^{\prime}, \xi^{\prime}\right\rangle(x) .
\end{aligned}
$$

Comme $\xi$ et $\xi^{\prime}$ sont totalisateurs pour $\pi$ et $\pi^{\prime}$, il existe (lemme 2.5) un unique unitaire $U$ de $\mathcal{E}$ sur $\mathcal{E}^{\prime}$ vérifiant $U \pi(f)=\pi^{\prime}(f) U$ et $U_{s(\gamma)} \xi_{s(\gamma)}=\xi_{s(\gamma)}^{\prime}$ pour tout $f \in L^{1}(G, \lambda)$. La relation $U \pi(f)=\pi^{\prime}(f) U$ implique

$$
\left\langle\pi(f) \xi, U^{*} \xi^{\prime}\right\rangle(x)=\left\langle\pi^{\prime}(f) U \xi, \xi^{\prime}\right\rangle(x)
$$

Donc

$$
\int_{\gamma \in G^{x}}\left\langle\pi_{\gamma} \xi_{s(\gamma)}, U_{r(\gamma)}^{*} \xi_{r(\gamma)}^{\prime}\right\rangle f(\gamma) d \lambda^{x}(\gamma)=\int_{\gamma \in G^{x}}\left\langle\pi_{\gamma}^{\prime} U_{s(\gamma)} \xi_{s(\gamma)}, \xi_{r(\gamma)}^{\prime}\right\rangle f(\gamma) d \lambda^{x}(\gamma)
$$

pout tout $f \in L^{1}(G, \lambda)$. Par conséquent $U_{r(\gamma)}^{*} \pi_{\gamma}^{\prime} U_{s(\gamma)}=\pi_{\gamma}$. 
Démonstration de la proposition (2.3).

Pour $\xi \in C_{c}\left(G_{2}\right)$, on pose

$$
\varphi\left(g_{1}\right)=\left\langle\lambda^{G_{2}}\left(f\left(g_{1}\right)\right) \xi_{s\left(f\left(g_{1}\right)\right)}, \xi_{r\left(f\left(g_{1}\right)\right)}\right\rangle .
$$

Comme $f$ est propre, la fonction $\varphi$ est de type positif et à support compact. D'après [1] (lemme 2.2.10), il existe $\xi^{\prime} \in C_{c}\left(G_{1}\right)$ tel que

$$
\varphi\left(g_{1}\right)=\left\langle\lambda^{G_{2}}\left(f\left(g_{1}\right)\right) \xi_{s\left(f\left(g_{1}\right)\right)}, \xi_{r\left(f\left(g_{1}\right)\right)}\right\rangle=\left\langle\lambda^{G_{1}}\left(g_{1}\right) \xi_{s\left(g_{1}\right)}^{\prime}, \xi_{r\left(g_{1}\right)}^{\prime}\right\rangle .
$$

D'après le lemme (2.6), $\lambda^{G_{2}} \circ f \subset \lambda^{G_{1}}$. Soit maintenant $F_{2} \in \mathcal{L}\left(\mathcal{H}_{G_{2}}\right)^{(1)}$ tel que $\left(\mathcal{H}_{G_{2}}, F_{2}\right)$ soit de classe 1 dans $K K_{G_{2}}\left(C\left(X_{2}\right), C\left(X_{2}\right)\right)$. Alors $f^{*}\left(\left(\mathcal{H}_{G_{2}}, F_{2}\right)\right)=\left(\mathcal{H}_{G_{2}}, F_{1}\right)=1$ dans $K K_{G_{1}}\left(C\left(X_{1}\right), C\left(X_{1}\right)\right)$.

Soit $\mathcal{G}$ un groupoïde de base $X$ agissant à gauche sur un ensemble $Z$. Soit $G=\mathcal{G} \times{ }_{s} Z=\left\{(h, x) \in \mathcal{G} \times Z: s(h)=r_{z}(x)\right\}$ le produit croisé de $\mathcal{G}$ par $Z$ où $r_{z}: Z \longrightarrow X$ est l'action de $\mathcal{G}$ sur $Z$. Rappelons que deux éléments $(h, x)$ et $\left(h^{\prime}, y\right)$ de $\mathcal{G} \times{ }_{s} Z$ sont composables si $x=h^{\prime} y$. On a $\left(h, h^{\prime} y\right) \circ\left(h^{\prime}, y\right)=\left(h h^{\prime}, y\right)$ et $(h, x)^{-1}=\left(h^{-1}, h x\right)$. L'espace des unités de $G$ est $Z$. Si $\lambda=\left\{\lambda^{x}, x \in X\right\}$ est un système de Haar pour $\mathcal{G}$, la famille $\lambda^{\prime}=\left\{\lambda^{r_{z}(z)}, z \in Z\right\}$ définit un système de Haar pour $\mathcal{G} \times{ }_{s} Z$.

Proposition 2.7. - Soient $\mathcal{G}$ un groupoïde localement compact muni d'un sytème de Haar et $Z$ un $\mathcal{G}$-espace localement compact. Si $\mathcal{G}$ est fortement $K$-moyennable, alors le groupoïde $G=\mathcal{G} \times{ }_{s} Z$ est fortement $K$ moyennable.

Démonstration. - L'application $p$ de $G$ dans $\mathcal{G}$ qui à $(h, x)$ associe $h$ définit un homomorphisme de groupoïdes dont la restriction à $Z$ est $r_{z}$. Soient $p^{*}: K K_{\mathcal{G}}(C(X), C(X)) \longrightarrow K K_{G}(C(Z), C(Z))$ l'homomorphisme induit par $p$ [7] et $\left(\mathcal{H}_{\mathcal{G}}, F\right)$ le $\mathcal{G}$-module de Fredholm définissant la forte $K$ moyennabilité de $\mathcal{G}$. Donc $\left(\mathcal{H}_{\mathcal{G}}, F\right)=1$ dans $K K_{\mathcal{G}}(C(X), C(X))$. Puisque $L^{2}(\mathcal{G}, \lambda) \otimes_{C(X)} C(Z)$ est un sous- $C(Z)$-module hilbertien $G$-invariant de $L^{2}\left(G, \lambda^{\prime}\right)$, il existe alors $F^{\prime} \in \mathcal{L}\left(\mathcal{H}_{G}\right)^{(1)}$ tel que $p^{*}\left(\left[\left(\mathcal{H}_{\mathcal{G}}, F\right)\right]\right)=\left[\left(\mathcal{H}_{G}, F^{\prime}\right)\right]=$ 1.

\section{Suites exactes.}

Nous commençons cette section en rappelant la définition d'un bimodule $C(X)$-nucléaire (cf.[15],[12],[3]) et celle du bifoncteur $K K_{G}^{n u c}$ pour un groupoïde $G$ localement compact. Toutes les propriétés de ce bifoncteur étudiées dans $([15],[12])$ se généralisent facilement à ce cadre. 
DÉFINITION 3.1 ([15],[3]). - Soient $X$ un espace localement compact, $A$ et $B$ des $C^{*}$-algèbres avec une structure de $C(X)$-module. Soit $\varphi: A \longrightarrow$ $B$ une application complètement positive $C(X)$-linéaire.

1) On dit que $\varphi$ est $C(X)$-factorisable si elle admet une factorisation $\varphi=\sigma \circ \tau$ où $\tau: A \longrightarrow M_{n}(C(X))$ et $\sigma: M_{n}(C(X)) \longrightarrow B$ sont des applications complètement positives.

2) On dit que $\varphi$ est (normiquement) $C(X)$ - nucléaire, si $\varphi$ est limite pour la topologie simple normique d'applications complètement positives $C(X)$ factorisables.

3) Si $B=M(J)$ est l'algèbre des multiplicateurs d'une $C^{*}$-algèbre $J$, alors $\varphi$ est dite strictement $C(X)$-nucléaire si elle est limite pour la topologie simple stricte d'applications complètement positives $C(X)$-factorisables.

On suppose désormais que $A$ et $B$ sont des $C(X)$-algèbres [10]. Rappelons que pour tout $C^{*}$-module hilbertien $\mathcal{E}$, on a: $\mathcal{L}(\mathcal{E})=M(\mathcal{K}(\mathcal{E})$ ) [9]. On dit qu'un $A, B$-bimodule hilbertien $\mathcal{E}$ est $C(X)$-nucléaire si l'action $\pi: A \longrightarrow \mathcal{L}(\mathcal{E})$ est strictement $C(X)$-nucléaire ([15], [3]).

Un $A, B$-bimodule hilbertien $\mathcal{E}$ est $C(X)$-nucléaire si, et seulement si, pour tout $\xi_{1}, \xi_{2}, \ldots, \xi_{n}$ dans $\mathcal{E}$ l'application: $a \mapsto\left\langle\xi_{i}, \pi(a) \xi_{j}\right\rangle$ de $A$ dans $M_{n}(B)$ est (normiquement) $C(X)$-nucléaire . Par conséquent un sous- $A, B$ bimodule $\mathcal{E}^{\prime}$ d'un $A, B$-bimodule $C(X)$-nucléaire $\mathcal{E}$ est aussi $C(X)$-nucléaire.

Si $J$ est un idéal bilatère de $B$ et si $\varphi: A \longrightarrow M(B / J)$ est une application contractante strictement $C(X)$-nucléaire, il existe $\varphi^{\prime}: A \longrightarrow M(B)$ une application contractante strictement $C(X)$-nucléaire telle que $\varphi=q \circ \varphi^{\prime}$ où $q: M(B) \longrightarrow M(B / J)$ est l'application quotient [3]. Notons aussi que pour $\mathcal{E}_{1}$ un $A, B$-bimodule hilbertien et $\mathcal{E}_{2}$ un $B, D$-module hilbertien, si $\mathcal{E}_{1}$ ou $\mathcal{E}_{2}$ est $C(X)$-nucléaire, le $A, D$-bimodule $\mathcal{E}_{1} \otimes_{B} \mathcal{E}_{2}$ est aussi $C(X)$-nucléaire [3].

On dit qu'un $A, B$-bimodule hilbertien $Z / 2 Z$-gradué est $C(X)$-nucléaire si le $A, B$-bimodule trivialement gradué sous-jacent est $C(X)$-nucléaire.

Si $A$ ou $B$ est $C(X)$-nucléaire (i.e. $i d_{A}$ ou $i d_{B}$ est $C(X)$-nucléaire), alors tout $A, B$-bimodule est $C(X)$-nucléaire.

La somme directe de deux $A, B$-bimodules $C(X)$-nucléaires est un $A, B$ bimodule $C(X)$-nucléaire. 
3.1. Le groupe $K K_{G}^{n u c}(A, B)$.

Soit $G$ un groupoïde localement compact(séparé et séparable) de base $X$ et soient $A$ et $B$ deux $G$-algèbres ( $Z / 2 Z$-graduées).

DÉfinition 3.2.- 1) On note $E_{G}^{\text {nuc }}(A, B)$ l'ensemble des $A, B$ bimodules de Kasparov $(\mathcal{E}, F)$ avec $\mathcal{E}$ dénombrablement engendré et $C(X)$ nucléaire.

2) $D_{G}^{n u c}(A, B)=E_{G}^{n u c}(A, B) \cap D_{G}(A, B)$.

3) Une homotopie est un élément de $E_{G}^{n u c}(A, B[0,1])$.

4) On note $K K_{G}^{n u c}(A, B)$ le groupe des classes d'homotopie d'éléments de $E_{G}^{\text {nuc }}(A, B)$.

Si $A$ ou $B$ est $C(X)$-nucléaire, alors $K K_{G}^{n u c}(A, B)$ est égal à $K K_{G}(A, B)$. Une $G$-algèbre $A$ est $C(X)$-nucléaire en $K$-théorie équivariante si l'élément $1_{A} \in K K_{G}(A, A)$ est représenté par un $A, A$-bimodule de Kasparov $C(X)$ nucléaire. Si $A$ est $C(X)$-nucléaire en $K$-théorie équivariante, alors pour toute $G$-algèbre $B$, on a $K K_{G}^{n u c}(A, B)=K K_{G}(A, B)$ et $K K_{G}^{n u c}(B, A)=$ $K K_{G}(B, A)$.

Comme dans le cas d'un groupe nous avons:

THÉORÈME 3.3. - Soient $G$ un groupoïde localement compact, muni d'un système de Haar, fortement $K$-moyennable et $0 \longrightarrow$ $J \stackrel{j}{\longrightarrow} B \stackrel{q}{\longrightarrow} B / J \longrightarrow 0$ une suite exacte $G$-équivariante de $G$-algèbres. Pour toute $G$-algèbre séparable $A$, on a la suite exacte hexagonale suivante:

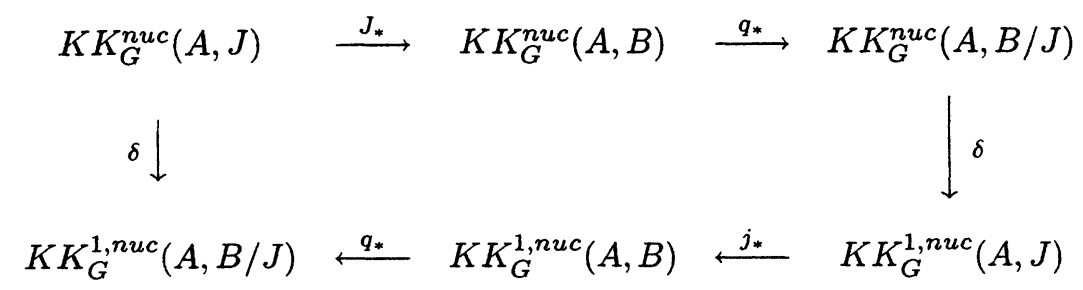

THÉORÈME 3.4. - Soit $G$ un groupoïde localement compact, muni d'un système de Haar, fortement $K$-moyennable. Soit $0 \rightarrow J \stackrel{\jmath}{\longrightarrow} B \stackrel{q}{\longrightarrow} B / J \rightarrow$ 0 une suite exacte $G$-équivariante de $G$-algèbres telle que $q$ admette un relèvement complètement positif $C(X)$-linéaire (non nécessairement équivariant) de norme 1. 
a) Si $A$ est séparable, on a la suite exacte hexagonale:

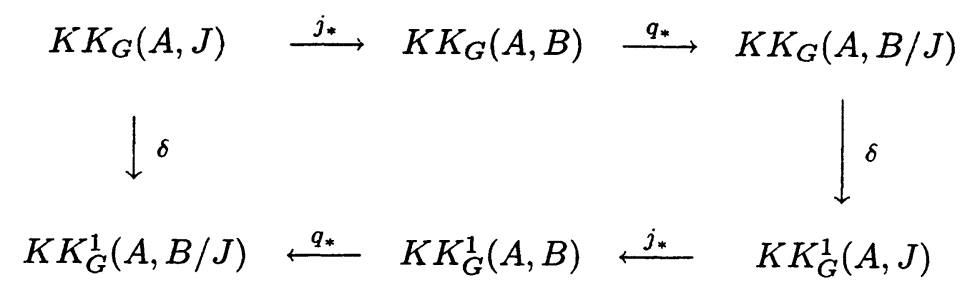

b) Si B est séparable, alors pour toute $G$-algèbre $A$, on a la suite exacte:

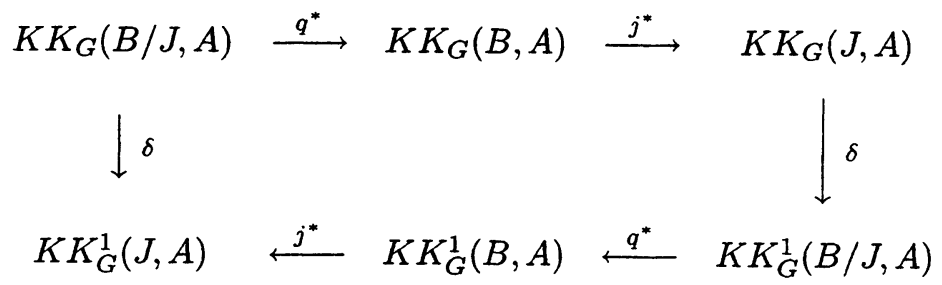

En utilisant les suites exactes de Puppe ([2], [6], [12], [17]) les théorèmes deviennent un corollaire du lemme suivant.

LEMME 3.5. - 1) Sous les hypothèses a) théorème (3.4), on a la suite exacte

$$
K K_{G}(A, J) \stackrel{j_{*}}{\longrightarrow} K K_{G}(A, B) \stackrel{q_{*}}{\longrightarrow} K K_{G}(A, B / J) .
$$

2) Sous les hypothèses du théorème (3.3), on a la suite exacte suivante:

$$
K K_{G}^{n u c}(A, J) \stackrel{j_{*}}{\longrightarrow} K K_{G}^{n u c}(A, B) \stackrel{q_{*}}{\longrightarrow} K K_{G}^{n u c}(A, B / J) .
$$

Tous les lemmes intermédiaires ayant servi à la démonstration de ce lemme dans le cas d'un groupe se généralisent sans aucune difficulté à ce cadre. Nous les rappelons en donnant des esquisses des démonstrations. Pour plus de detail voir $([14],[12],[16],[17])$.

LEMME 3.6. - Soit $(\mathcal{E}, F)$ un élément de $E_{G}(A, B)$ tel que $q_{*}(\mathcal{E}, F) \in$ $D_{G}(A, B / J)$. Il existe alors $\left(\mathcal{E}^{\prime}, F^{\prime}\right) \in E_{G}(A, J)$ tel que $j_{*}\left(\mathcal{E}^{\prime}, F^{\prime}\right)=(\mathcal{E}, F)$ 
dans $K K_{G}(A, B)$. Si de plus $\mathcal{E}$ est $C(X)$-nucléaire, alors $\left(\mathcal{E}^{\prime}, F^{\prime}\right)$ l'est et l'égalité précédente a lieu dans $K K_{G}^{n u c}(A, B)$.

Démonstration. - Si on pose $\mathcal{E}^{\prime}=\{\xi \in \mathcal{E} /\langle\xi, \xi\rangle \in J\}$ et $\tilde{\mathcal{E}}=\{\xi \in$ $\left.\mathcal{E}[0,1] / \xi(1) \in \mathcal{E}^{\prime}\right\}$. Alors $(\tilde{\mathcal{E}}, F \otimes 1)$ définit une homotopie entre $(\mathcal{E}, F)$ et $\left(\mathcal{E}^{\prime}, F^{\prime}\right) \in j_{*}\left(E_{G}(A, J)\right)$.

Si de plus $\mathcal{E}$ est $C(X)$ - nucléaire, alors $\mathcal{E}^{\prime}$ et $\tilde{\mathcal{E}}$ sont aussi $C(X)$-nucléaires ([12] proposition 3.4).

Dans la suite, on notera $a x=\pi(a) x$ et $V$ l'unitaire représentant l'action du groupoïde $G$. Pour le reste de la notation voir([14],[12],[17],,[7]).

LeMme 3.7. - Soit $(\mathcal{E}, \pi)$ un $A, B$-bimodule équivariant. On pose

$\mathcal{A}=\left\{x \in \mathcal{L}(\mathcal{E}) /[a, x] \in \mathcal{K}(\mathcal{E}) \quad \forall a \in A\right.$ et $a^{\prime}\left(V\left(s^{*} x\right) V^{*}-r^{*} x\right) \in$ $\left.r^{*} \mathcal{K}(\mathcal{E}), \forall a^{\prime} \in r^{*} A\right\}$

$\mathcal{A}^{\prime}=\left\{x \in \mathcal{L}\left(\mathcal{E} \otimes_{q} B / J\right) /[a, x] \in \mathcal{K}\left(\mathcal{E} \otimes_{q} B / J\right) \forall a \in A\right.$ et $a^{\prime}\left(V\left(s^{*} x\right) V^{*}-\right.$ $\left.\left.r^{*} x\right) \in r^{*} \mathcal{K}\left(\mathcal{E} \otimes_{q} B / J\right), \quad \forall a^{\prime} \in r^{*} A\right\}$

$$
\begin{aligned}
& \mathcal{G}=\{x \in \mathcal{A} / x a \in \mathcal{K}(\mathcal{E}), \quad \forall a \in A\} \\
& \mathcal{G}^{\prime}=\left\{x \in \mathcal{A}^{\prime} / x a \in \mathcal{K}\left(\mathcal{E} \otimes_{q} B / J\right), \quad \forall a \in A\right\} \\
& \mathcal{D}=\mathcal{A} / \mathcal{G} \text { et } \mathcal{D}^{\prime}=\mathcal{A}^{\prime} / \mathcal{G}^{\prime} .
\end{aligned}
$$

Alors l'homomorphisme $q_{*}: \mathcal{L}(\mathcal{E}) \longrightarrow \mathcal{L}\left(\mathcal{E} \otimes_{B} B / J\right)\left(q_{*}(x)=x \otimes 1\right)$ induit un homomorphisme surjectif de $\mathcal{D}$ dans $\mathcal{D}^{\prime}$.

Démonstration. - La démonstration de Baaj et Skandalis ([2] lemme 7.4) dans le cas d'un groupe quantique s'adapte facilement (en utilisant le lemme technique de Kasparov dans le cas d'un groupoïde [7]) en remplaçant $E_{1} \otimes S$ par $r^{*} E_{1}, \quad V\left(x \otimes_{\delta_{B}} 1\right) V^{*}$ par $V\left(s^{*} x\right) V^{*}, \quad M\left(E_{1} \otimes S\right)$ par $E_{1} \otimes_{r} C_{b}(G)$ et $M\left(\tilde{M}\left(E_{1} \otimes S\right) ; E \otimes S\right)$ par $M\left(E_{1} ; E\right) \otimes_{r} C_{b}(G)$ etc....

Lemme 3.8. - Soient $(\mathcal{E}, F) \in E_{G}(A, B)$ et $\left(F_{t}\right)_{t \in[0,1]}$ une homotopie opératorielle telle que:

$$
F_{t} \in \mathcal{L}\left(\mathcal{E} \otimes_{B} B / J\right),\left(\mathcal{E} \otimes_{B} B / J, F_{t}\right) \in E_{G}(A, B / J) \text { et } F \otimes 1=F_{0} .
$$

Alors il existe une homotopie opératorielle $S_{t}$ avec $\left(\mathcal{E}, S_{t}\right) \in E_{G}(A, B)$, $S_{0}=F$ et $S_{t} \otimes 1-F_{t} \in \mathcal{K}\left(\mathcal{E} \otimes_{B} B / J\right)$.

Démonstration. - Soient $\varrho: \mathcal{A} \longrightarrow \mathcal{D}$ et $\varrho^{\prime}: \mathcal{A}^{\prime} \longrightarrow \mathcal{D}^{\prime}$ les applications canoniques (lemme 3.7). On a $F \in \mathcal{A}$ et $F_{t} \in \mathcal{A}^{\prime}$. On pose $f=\varrho(F)$ et 
$f_{t}=\varrho^{\prime}\left(F_{t}\right)$. Il existe (voir [14], [12]) $\left(s_{t}\right)_{t \in[0,1]}$ dans $\mathcal{D}$ telle que:

$$
q_{*}\left(s_{t}\right)=f_{t}, s_{t}^{2}=1, s_{t}=s_{t}^{*}, V\left(s_{t} \otimes 1\right) V^{*}=r^{*} s_{t}, \partial s_{t}=1, s_{0}=f .
$$

Soit $\left(S_{t}\right)_{t \in[0,1]}$ un chemin continu dans $\mathcal{A}$ tel que $\varrho\left(S_{t}\right)=s_{t}$ et $S_{0}=F$. Par définition de $q$, on a $S_{t} \otimes 1-F_{t} \in \mathcal{K}\left(\mathcal{E} \otimes_{B} B / J\right)$.

Soient $A, B$ deux $C^{*}$-algèbres, $\mathcal{E}$ un $B$-module hilbertien et $\varphi: \tilde{A} \longrightarrow$ $\mathcal{L}(\mathcal{E})$ une application complètement positive avec $\varphi(1)=1$. Généralisant un théorème de Stinespring, Kasparov a construit un $B$-module hilbertien $\tilde{\mathcal{E}}$, une isométrie $W \in \mathcal{L}(\mathcal{E}, \tilde{\mathcal{E}})$ et un $*$-homomorphisme $\pi: A \longrightarrow \mathcal{L}(\tilde{\mathcal{E}})$ avec $\varphi(a)=W^{*} \pi(a) W$. Rappelons que $\tilde{\mathcal{E}}$ est le séparé complété du produit tensoriel algébrique $A \otimes_{\text {alg }} \mathcal{E}$ pour le produit scalaire:

$$
\left\langle\sum_{i=1}^{n} a_{i} \otimes x_{i}, \sum_{j=1}^{m} a_{j} \otimes x_{j}\right\rangle=\sum_{i=1}^{n} \sum_{j=1}^{m}\left\langle x_{i}, \varphi\left(a_{i}^{*} a_{j}\right) x_{j}\right\rangle
$$

l'homomorphisme $\pi$ est donné par multiplication à gauche et $W \xi=1 \otimes \xi$. Si $\varphi$ est un $*$-homomorphisme alors $\tilde{\mathcal{E}}=\tilde{A} \otimes_{\tilde{A}} \mathcal{E}=\mathcal{E}$.

Si l'application $\varphi$ ci-dessus est strictement $C(X)$ - nucléaire, alors il en est de même pour $\pi$ ([3] proposition 4.4).

Remarque. - Si $\varphi$ est le relèvement d'un homomorphisme $\varphi^{\prime}: A \longrightarrow$ $\mathcal{L}\left(\mathcal{E} \otimes_{q} B / J\right)$ i.e. $\varphi^{\prime}=\varphi \otimes 1$, alors grâce à l'associativité du produit tensoriel interne, on a $q \circ \pi=\pi \otimes 1=\varphi^{\prime}$.

Lemme 3.9. - Soit $G$ un groupoïde localement compact de base $X$ et muni d'un système de Haar $\lambda$. Soient $A$ une $G$-algèbre, $\mathcal{E}$ un $B$-module hilbertien $G$-équivariant et $\pi: A \longrightarrow \mathcal{L}(\mathcal{E})$ une application complètement positive $C(X)$-linéaire. Alors, l'application: $\bar{\pi}$ de $A$ dans $\mathcal{L}\left(L^{2}(G, \mathcal{E})\right)$ donnée par: $\bar{\pi}(a) f\left(\gamma^{\prime}\right)=\tilde{V}_{\gamma^{\prime}}\left(\pi_{s\left(\gamma^{\prime}\right)}\left(\alpha_{\gamma^{\prime-1}} a_{r\left(\gamma^{\prime}\right)}\right)\right) f\left(\gamma^{\prime}\right)$ est complètement positive, $C(X)=$ =linéaire et équivariante où $\tilde{V}_{\gamma}: \mathcal{L}\left(\mathcal{E}_{s(\gamma)}\right) \longrightarrow \mathcal{L}\left(\mathcal{E}_{r(\gamma)}\right)$ est tel que: $\tilde{V}_{\gamma}(T)(\xi)=V_{\gamma}\left(T\left(V_{\gamma}^{*} \xi\right)\right)$ et $V$ est l'unitaire associé à l'action de $G$ sur $\mathcal{E}$.

Démonstration. - Il est facile de voir que $\bar{\pi}$ est continue, complètement positive et $C(X)$-linéaire. Vérifions alors l'équivariance. Rappelons que l'action de $G$ sur $L^{2}(G, \mathcal{E})$ est donnée par: $V_{\gamma}^{\prime}(f)\left(\gamma^{\prime}\right)=V_{\gamma}\left(f\left(\gamma^{-1} \gamma^{\prime}\right)\right)$. Pour tous $\gamma \in G$ et $a \in A_{s(\gamma)}$, on a: 


$$
\begin{aligned}
{\left[\left[V_{\gamma}^{\prime} \bar{\pi}_{s(\gamma)}(a) V_{\gamma}^{\prime *}\right] f\right]\left(\gamma^{\prime}\right) } & =V_{\gamma}^{\prime}\left[\bar{\pi}_{s(\gamma)}(a)\left(V_{\gamma}^{\prime *}(f)\right)\right]\left(\gamma^{\prime}\right) \\
& =V_{\gamma}\left[\left[\bar{\pi}_{s(\gamma)}(a)\left(V_{\gamma}^{\prime *}(f)\right)\right]\left(\gamma^{-1} \gamma^{\prime}\right)\right] \\
& =V_{\gamma}\left[\left[\tilde{V}_{\gamma}^{*} \tilde{V}_{\gamma^{\prime}}\left(\pi_{s\left(\gamma^{\prime}\right)}\left(\alpha_{\gamma^{\prime-1} \gamma} a\right)\right)\right]\left(V_{\gamma}^{*}\left(f\left(\gamma^{\prime}\right)\right)\right)\right] \\
& =V_{\gamma}\left[\left[V_{\gamma}^{*} V_{\gamma^{\prime}}\left(\pi_{s\left(\gamma^{\prime}\right)}\left(\alpha_{\gamma^{\prime-1} \gamma} a\right)\right) V_{\gamma}^{*} V_{\gamma}\right]\left(V_{\gamma}^{*}\left(f\left(\gamma^{\prime}\right)\right)\right)\right] \\
& =V_{\gamma^{\prime}}\left[\pi_{s\left(\gamma^{\prime}\right)}\left(\alpha_{\gamma^{\prime-1} \gamma} a\right)\left(V_{\gamma^{\prime}}^{*}\left(f\left(\gamma^{\prime}\right)\right)\right)\right] \\
& =\left[\tilde{V}_{\gamma^{\prime}}\left(\pi_{s\left(\gamma^{\prime}\right)}\left(\alpha_{\gamma^{\prime-1} \gamma} a\right)\right)\right](f)\left(\gamma^{\prime}\right) \\
& \left.=\left[\bar{\pi}_{r(\gamma)}\left(\alpha_{\gamma} a\right)\right) f\right]\left(\gamma^{\prime}\right) .
\end{aligned}
$$

Si de plus $\pi$ est une limite pour la topologie simple stricte d'applications $C(X)$-factorisables $\pi_{k}$, alors $\bar{\pi}_{k}$ converge pour la topologie simple stricte vers $\bar{\pi}$ car l'action de $G$ dans $\mathcal{L}(\mathcal{E})=M(\mathcal{K}(\mathcal{E})$ ) est continue pour la topologie stricte.

Soit $H^{\prime}=l^{2}(\mathbb{N}) \oplus l^{2}(\mathbb{N})$ et $\varepsilon \in \mathcal{L}\left(H^{\prime}\right)$ tel que $\partial \varepsilon=1, \quad \varepsilon=\varepsilon^{*}, \quad \varepsilon^{2}=1$. Soient $\mathcal{C}_{1}=\boldsymbol{C}+\varepsilon \boldsymbol{C} \subseteq \mathcal{L}\left(H^{\prime}\right)$ la première algèbre de Clifford et $\mathcal{H}_{G}=$ $L^{2}(G) \otimes H^{\prime}$.

Lemme 3.10. - Soit $(\mathcal{E}, F)$ un élément de $D_{G}^{\text {nuc }}(A, B / J)$. Il existe alors $\left(\mathcal{E}^{\prime}, F^{\prime}\right) \in D_{G}^{n u c}(A, B)$ tel que $q_{*}\left(\mathcal{E}^{\prime}, F^{\prime}\right)=\left(\mathcal{H}_{G} \otimes_{C(X)} \mathcal{E}, 1 \otimes F\right)$.

Démonstration. - Soit $\varphi: A \otimes \mathcal{C}_{1} \longrightarrow \mathcal{L}\left(\mathcal{H}_{B / J}\right)$ l'*-homomorphisme équivariant strictement $C(X)$-nucléaire associé à $(\mathcal{E}, F) \in D_{G}^{n u c}(A, B / J)$ $\left(\varphi(a \otimes(\mu+\lambda \varepsilon)=a(\mu+\lambda F))\right.$. Prolongeons $\varphi$ à $\tilde{A} \otimes \mathcal{C}_{1}$ en posant $\varphi(1)=1$. On obtient donc un *-homomorphisme unital strictement $C(X)$-nucléaire de $\tilde{A} \otimes$ $C_{1}$ dans $\mathcal{L}\left(\mathcal{H}_{B / J}\right)$ qu'on notera encore $\varphi$. Il existe $\psi: \tilde{A} \otimes \mathcal{C}_{1} \longrightarrow \mathcal{L}\left(\mathcal{H}_{B}\right)$ une application complètement positive strictement $C(X)$-nucléaire qui relève $\varphi$. Soit $\tilde{\psi}$ l'homomorphisme strictement $C(X)$-nucléaire associé à $\psi$ par le lemme (3.8). Identifions $\mathcal{H}_{G} \otimes_{C(X)} \mathcal{H}_{B}$ à $L^{2}\left(G, \mathcal{H}_{B}\right)$. L'homomorphisme

$$
\bar{\psi}: \tilde{A} \otimes \mathcal{C}_{1} \longrightarrow \mathcal{L}\left(\mathcal{H}_{G} \otimes_{C(X)} \mathcal{H}_{B}\right)
$$

donné par:

$$
\bar{\psi}(a) f\left(\gamma^{\prime}\right)=\tilde{V}_{\gamma^{\prime}}\left(\tilde{\psi}_{s\left(\gamma^{\prime}\right)}\left(\alpha_{\gamma^{\prime-1}} a_{r\left(\gamma^{\prime}\right)}\right)\right) f\left(\gamma^{\prime}\right)
$$

définit un relèvement équivariant strictement $C(X)$-nucléaire de $1 \otimes \varphi$ (lemmes 3.8 et 3.9$)$. L'élément $\left(\mathcal{E}^{\prime}, F^{\prime}\right)$ cherché est donné par la restriction de $\bar{\psi}$ à $A \otimes \mathcal{C}_{1}$.

LEMME 3.11. - Soit $0 \longrightarrow J \stackrel{j}{\longrightarrow} B \stackrel{q}{\longrightarrow} B / J \longrightarrow 0$ une suite exacte de $G$ algèbres telle que $q$ admette un relèvement complètement positif. Soient $A$ 
une $G$-algèbre séparable et $(\mathcal{E}, F)$ un élément de $D_{G}(A, B / J)$. On pose $\tilde{\mathcal{E}}=$ $\mathcal{H}_{G} \otimes_{C(X)} \mathcal{E}$ et $\tilde{F}=1 \otimes F$. Il existe alors $\left(\mathcal{E}^{\prime}, F^{\prime}\right) \in D_{G}(A, B)$ tel que $q_{*}\left(\mathcal{E}^{\prime}, F^{\prime}\right)=(\tilde{\mathcal{E}}, \tilde{F})$.

Démonstration. - Par le théorème 3 [9], il existe un $B$-module hilbertien $\mathcal{E}_{0}$ et un $*$-homomorphisme $\pi: B / J \longrightarrow \mathcal{L}\left(B \oplus \mathcal{E}_{0}\right)$ tels que $Q \pi(b) Q=s(b)$, pour tout $b \in B$ où $Q: B \oplus \mathcal{E}_{0} \longrightarrow B$ est la projection naturelle et $s$ est le relèvement de $q$. Notons $\overline{\mathcal{E}}=\mathcal{H}_{G} \otimes_{C(X)}\left(B \oplus \mathcal{E}_{0}\right)$ et $\bar{\pi}: B / J \longrightarrow \mathcal{L}(\overline{\mathcal{E}})$ l'application donnée par $\bar{\pi}(a) f\left(\gamma^{\prime}\right)=\tilde{V}_{\gamma^{\prime}}\left(\pi_{s\left(\gamma^{\prime}\right)}\left(\alpha_{\gamma^{\prime-1}} a_{r\left(\gamma^{\prime}\right)}\right)\right) f\left(\gamma^{\prime}\right)$. Alors $\bar{\pi}$ est un $*$-homomorphisme équivariant (lemme 3.9). Posons $\mathcal{E}^{\prime}=\mathcal{E} \otimes_{B / J} \overline{\mathcal{E}}$ et $F^{\prime}=F \otimes 1$. On vérifie facilement que $\left(\mathcal{E}^{\prime}, F^{\prime}\right)$ est un élément de $D_{G}(A, B)$ et $q_{*}\left(\mathcal{E}^{\prime}, F^{\prime}\right)=(\tilde{\mathcal{E}}, \tilde{F})$.

Démonstration du lemme (3.5).

- Il est évident que $q_{*} \circ j_{*}=0$ dans chacune des deux suites 1) et 2).

- Montrons pour les deux suites 1) et 2) que $\operatorname{Ker} q_{*} \subset \operatorname{im} j_{*}$.

Soit $(\mathcal{E}, F) \in E_{G}(A, B)$ (resp. $\in E_{G}^{\text {nuc }}(A, B)$ ) tel que $q_{*}(\mathcal{E}, F)=(0,0)$ dans $K K_{G}(A, B / J)$ (resp. $K K_{G}^{n u c}(A, B / J)$ ). Soit $T \in \mathcal{L}\left(\mathcal{H}_{G}\right)^{(1)}$ un opérateur définissant la forte en $K$-moyennnabilité de $G$. Rappelons que $\left(\mathcal{H}_{G}, T\right)=1$ dans $K K_{G}(C(X), C(X))$. Soit $\tilde{F} \in \mathcal{L}\left(\mathcal{H}_{G} \otimes_{C(X)} \mathcal{E}\right)$ un produit de Kasparov de $\left(\mathcal{H}_{G}, T\right)$ par $(\mathcal{E}, F)$. Il existe donc $\left(\mathcal{E}_{0}, F_{0}\right)$ et $\left(\mathcal{E}_{1}, F_{1}\right)$ deux éléments de $D_{G}(A, B / J)$ (resp. de $D_{G}^{\text {nuc }}(A, B / J)$ ) tels que $q_{*}\left(\mathcal{H}_{G} \otimes_{C(X)} \mathcal{E}, \tilde{F}\right) \oplus\left(\mathcal{H}_{G} \otimes_{C(X)} \mathcal{E}_{0}, 1 \otimes F_{0}\right)$ soit opératoriellement homotope à $\left(\mathcal{H}_{G} \otimes_{C(X)} \mathcal{E}_{1}, 1 \otimes F_{1}\right)$. D'après le lemme (3.11) (resp. lemme (3.10)), il existe $\left(\mathcal{E}_{0}^{\prime}, F_{0}^{\prime}\right) \in D_{G}(A, B)$ (resp. $\in D_{G}^{n u c}(A, B)$ ) tel que $q_{*}\left(\mathcal{E}_{0}^{\prime}, F_{0}^{\prime}\right)=$ $\left(\mathcal{H}_{G} \otimes_{C(X)} \mathcal{E}_{0}, 1 \otimes F_{0}\right)$. Donc

$$
q_{*}\left(\left(\mathcal{H}_{G} \otimes_{C(X)} \mathcal{E} \oplus \mathcal{E}_{0}^{\prime}, \tilde{F} \oplus F_{0}^{\prime}\right)\right) \text { et }\left(\mathcal{H}_{G} \otimes_{C(X)} \mathcal{E}_{1}, 1 \otimes F_{1}\right)
$$

sont opératoriellement homotopes.

Notons par $\left(S_{t}\right)_{t \in[0.1]}$ cette homotopie opératorielle. Il existe d'après le lemme (3.8) une homotopie opératorielle $\left(G_{t}\right)_{t \in[0,1]}$ telle que $\left(\mathcal{H}_{G^{\prime}} \otimes C^{\prime}(X) \mathcal{E} \oplus\right.$ $\left.\mathcal{E}_{0}^{\prime}, G_{t}\right) \in E_{G}(A, B)\left(\right.$ resp. $\left.\in E_{G}^{n u c}(A, B)\right), G_{0}=\tilde{F} \oplus F_{0}^{\prime}$ et $G_{1} \otimes 1=1 \otimes F_{1}$. L'élément $\left(\mathcal{H}_{G} \otimes \mathcal{E}_{1}, 1 \otimes F_{1}\right)$ est dégénéré. D'après le lemme (3.6), la classe de $\left(\mathcal{H}_{G} \otimes \mathcal{E} \oplus \mathcal{E}_{0}^{\prime}, G_{1}\right)$ dans $K K_{G}(A, B)$ (resp. dans $\left.K K_{G}^{n u c}(A, B)\right)$ est dans l'image de $j_{*}$. Cornme $\left(\mathcal{H}_{G}, T\right)$ est de classe 1 dans $K K_{G}(C(X), C(X))$ et que la classe de $(\mathcal{E}, F)$ dans $K K_{G}(A, B)$ (resp. dans $K K_{G}^{\text {nuc }}(A, B)$ ) ne change pas par addition d'un élément dégénéré, le lemme (3.5) est donc démontré. 
Remarques. - 1) La moyennabilité forte en $K$-théorie est utilisée à deux reprises dans la démonstration du lemme (3.5):

- D'abord dans le choix de $\mathcal{H}_{G}$; ceci nous permet de remplacer $\left(\mathcal{E}_{0}, F_{0}\right)$ par $\left(\mathcal{H}_{G} \otimes_{C(X)} \mathcal{E}_{0}, 1 \otimes F_{0}\right)$ qui est relevable en un élément de $D_{G}(A, B)$ (resp. de $D_{G}^{\text {nuc }}(A, B)$ ).

- D'autre part, parce que $\left(\mathcal{H}_{G}, T\right)=1$ dans $K K_{G}(C(X), C(X))$, le produit de Kasparov de $(\mathcal{E}, F)$ par $\left(\mathcal{H}_{G}, T\right)$ définit le même élément que $(\mathcal{E}, F)$ dans $K K_{G}(A, B)$ (resp. dans $K K_{G}^{n u c}(A, B)$ ).

2) Le théorème 3.4 a été établi dans [16] mais dans le cas où il existe un $G$-espace propre $Z$ tel que $B / J$ soit une $Z \times G$-algèbre.

\section{Bibliographie}

[1] Anantharaman-Delaroche (C.) et Renault (J.), 1998. - Preprint Université d'Orléans.

[2] BaAJ (S.) et Skandalis (G.), 1989. $-C^{*}$-algèbres de Hopf et théorie de Kasparov équivariante, $K$-theory. 2 , p. 683-721.

[3] Bauval (A.), 1998. - $R K K(X)$-nucléarité (D'après G. Skandalis), $K$-théory. 13, p 23-40.

[4] Dixmier (J.), 1969. - Les $C^{*}$-algèbres et leurs représentations, Gauthier-Villars Paris.

[5] Cuntz (J.), 1983. $-K$-theoretic amenability for discrete groups. J. Reine Angew. Math. 344, p. 180-195.

[6] Cuntz (J.) et Skandalis (G.), 1986. - Mapping cones and exact sequences in $K K$ theory, J. Operator Theory. 15, p. 163-180.

[7] LE GALl (P-Y.), 1999. - Théorie de Kasparov équivariante et groupoïdes, $K$-theory. $16, \mathrm{p} / 361-390$.

[8] Julg (P.) et Valette (A.), 1984. $-K$-theoretic amenability for $S L_{2}(Q)$, and the action on the associated tree, J. Funct. Anal. 58, p194-215.

[9] Kasparov (G. G.) 1980. - Hilbert $C^{*}$-modules: theorems of Stinespring and Voiculescu, J. Operator Theory. 4, p. 133-150.

[10] KASPAROV (G.G.), 1988. - Equivariant $K K$-theory and the novikov conjecture. Invent. math. 91, p. 147-201.

[11] Kasparov (G.G.) et Skandalis (G.). - groups acting properly on " bolic " spaces and the Novikov conjecture, à paraitre.

[12] Maghfoul (M.), 1999. - Semi-exactitude du bifoncteur de Kasparov équivariant. $K$-theory. 16 p. $1-32$

[13] Renault (J.N.), 1980. - A groupoid approach to $C^{*}$-algebras. Springer Lecture Note in Math. 793.

[14] SkAndalis (G.), 1985. - Exact sequences for the Kasparov groups of graded algebras, Canadian J. Math. 37, p. 293-216. 
Sur la forte $K$-moyennabilité d'un groupoïde

[15] Skandalis (G.), 1988. - Une notion de nucléarité en $K$-théorie (d'après J. Cuntz). $K$-theory. 1, p. 549-573.

[16] TU (J.-L.), 1999. - La conjecture de Baum-Connes pour les feuilletages moyennables, $K$-theory. 17 , p 215-264.

[17] Tu (J.-L.), 1996. - La conjecture de Novikov pour les feuilletages hyperboliques, Thèse de Doctorat, Université Paris VII. 